\title{
D-dimer pitfalls and performance in COVID-19
}

Desempeño y artificios del dímero-D en COVID-19

Performance e artifícios de dimero-D em COVID-19

José Javier Elizalde González*

Since the first reports from China at the beginning of the COVID-19 pandemic, a high frequency of thrombotic phenomena in different vascular territories was described in these patients, particularly at the pulmonary level, although also in the deep venous system of the lower limbs (DVT), brain and heart among others, reaching in some cases to meet criteria of disseminated intravascular coagulation.

SARS-CoV-2 usually produces a significant thromboinflammatory response in the severe pneumonic forms of COVID-19 with progressive diffuse lung damage. The endothelial cells (ECs) play a significant role in vasodilation control, thrombotic regulation, fibrinolysis and antiaggregation between other important functions; with hypercoagulable profiles associated with severe disease indicating significant active SARS-CoV-2 ECs injury and dysfunction that can last for months in survivors, not to mention the havoc produced on alveolar epithelial cells, that will most likely trigger interstitial lung disease of different magnitude in the medium and long term, which could hit humanity in the near future particularly hard with thousands of new cases of progressive pulmonary fibrosis.

The highly thrombogenic basement membrane get exposed in the course of the disease, resulting in the pathological activation of the clotting cascade. Additionally, some proinflammatory substances as IL-1 $\beta$ and TNF activated ECs trigger the coagulation process by expressing P-selectin, von Willebrand factor and fibrinogen, to which platelets bind. In turn, ECs release trophic cytokines that further increase platelet production. Platelets also release vascular endothelial cell growth factor (VEGF), which activates ECs to upregulate the expression of the tissue factor, the main trigger of the coagulation cascade. The physiological response focuses on dissolution of the fibrin-rich blood clots, explaining why the D-dimers rise. D-dimer $>1,000$ has been associated with poor outcomes in the pandemic that we are currently suffering.

But what does this sometimes so important elevation of the D-dimer in severe patients with COVID-19 that we are seeing in the ICU really represent to the clini-

\footnotetext{
* Editor, INCMNSZ.
}

www.medigraphic.com/medicinacritica cian and how to interpret it? The answer is probably not simple as we are still learning and have only incomplete parts of the pathophysiology of the process, so that their treatment must be well reasoned.

As we know D-dimer is one of the proteins fragments and the main product of fibrin degradation by plasmin and is generated in the final phase of a clot formation; it is also known as fragment D-dimer and fibrin degradation fragment. It is normally undetectable or detectable at a very low level unless the body is forming and breaking down blood clots. Then, its level in the blood can significantly rise.

However as it is well known, it is sensitive but non specific of thrombosis and a number of other factors can cause an increased level. It's so that elevated concentrations may be seen in conditions in which fibrin is formed and then broken down, such as recent surgery, trauma, infection, sepsis, heart attack, pregnancy and some cancers or conditions in which fibrin is not cleared normally, such as liver disease and advanced age as well as in inflammatory diseases as rheumatoid arthritis. Therefore, D-dimer is typically not used to rule out venous thromboembolism (VTE) in hospitalized patients, and less in the critically ill who are perennially living in an inflammatory state.

However, in these times of adjustment in clinical medicine, all patients with severe forms of COVID-19 pneumonia usually undergo a series of laboratory studies that typically include the quantification of D-dimer, forming part of the «COVID-19 profile» in many labs, not always easy to interpret on a day-to-day basis, taking into account the dimension of the problem that is being measured, as well as the different assays to carry it out, quantitative, semiquantitative, qualitative rapid enzymelinked immunoabsorbent assays, quantitative and semiquantitave latex, and whole-blood assays; knowing that the quantitative rapid enzyme-linked immunoabsorbent assays are the best assays to use in terms of sensitivity and likelihood ratio so useful in the regular clinical arena.

When traditional clinical prediction scores indicate that the patient has a low or moderate pretest probability of pulmonary embolism (PE), D-dimer testing is useful to further define the likelihood of PE. Negative results on a high-sensitivity D-dimer test in a patient with a low pretest probability of PE (famous wells rule) are associated with a low likelihood of VTE and reliably exclude PE. 
The problem is that the pretest probability requires, regardless of experience in the specific problem, to be able to see the patient in the usual clinical way in medicine, to perform a complete physical exam and have reliable and accurate clinical data, especially in the critically ill. Medical practice at the moment has been involved in different improvised changes and adaptations in such a way that many complex decisions in critical care are being made remotely, based on second-hand information sometimes not fully verifiable by the most experienced ICU personnel.

In the past, large prospective, randomized trials in patients with a low probability of $P E$ and who had normal D-dimer levels, showed that not performing additional diagnostics testing was not associated with an increased frequency of symptomatic thromboembolic events during the subsequent six months; whether it is feasible to extrapolate these data to the current context of the intensely inflamed critically ill patient with severe pneumonic forms of COVID is unknown, but maybe not. D-dimer determination loses its ability to predict the likelihood of PE in many instances, as older age, active cancer, severe trauma, burns, pregnancy and various inflammatory and infectious diseases and disorders. It has been regular practice for years in patients with a high clinical pretest probability for PE, not to run a Ddimer which is difficult to interpret in this instance, generating more noise than answers and carry out more objective tests as a pulmonary angioCT scan and follow real time physiological parameters as the $\mathrm{PetCO}_{2}$ trend, as well as the $\mathrm{PetCO} / 2 / \mathrm{PaO}_{2}$ ratio.

This is probably the case in the vast majority of patients with severe COVID, who are being anticoagulated with full doses of heparin even without a certain diagnosis of PE or DVT, or sometimes receive new prophylactic «intermediate» doses of some LMWH, recommended in an informal way, without having scientific evidence on its safety and efficacy. We are aware of the development of bleeding complications in some cases, sometimes serious, although hard data will come in the following months and although small series of cases have been reported with lower mortality associated with the use of heparin, it is for now difficult to interpret this information, we just don't know.

But there is even greater uncertainty about the use of direct oral anticoagulants in severe patients with coro- navirus, none of the multiple and impeccable series of cases with dabigatran, rivaroxaban, apixaban or endoxaban, have been carried out with patients of these characteristics, therefore they should be prescribed with great caution and by a proper case by case analysis.

We know at least that LMWH have in addition to their known anticoagulant effect, another no less important such as an anti-inflammatory power.

There is evidence that venous thrombosis contributes to an active vein wall inflammatory response that is started and propagated by further thrombosis. This response is characterized by early vein wall neutrophil infiltration followed by monocyte and macrophage infiltration. Although the exact mechanism that governs the thrombosis/vein wall inflammatory response has not been fully elucidated, there appears to be a complex regulatory mechanism, involving a balance between pro-inflammatory and anti-inflammatory vein wall cytokines, that regulates the intensity of the inflammatory response. Concomitantly, platelet and endothelial cell derived adhesion molecules contribute to the initial reversible and subsequent irreversible influx and binding of these vein wall inflammatory cells to the endothelium, further propagating the inflammatory response. Regular heparin but especially LMWH possess clear anti-inflammatory properties distinct from its anticoagulant effects, and that is an advantage in patients with coronavirus severe inflammation associated with lung infection and ARDS.

The COVID-19 cytokinemia (IL-1B, IL-6, IL-8 and sTNFR1) is distinct from that of other types of pneumonia and other critical care situations, leading to organ failure, mechanical ventilation and ICU need. Neutrophils undergo immunometabolic reprogramming in severe COVID-19 illness, a complex reaction. So, what does elevated D-dimer levels really represent in severe COVID-19 pneumonia?, maybe only the tip of the iceberg of intricate thromboinflammation that clinicians can see, hopefully future well designed research will show us the reality.

Correspondence: José J. Elizalde González, M.D. E-mail: jjeg@unam.mx 